\title{
A Comparative Analysis of Optimum and Suboptimum Rake Receivers in Impulsive UWB Environment
}

\author{
Byoung Soo Kim, Jinsoo Bae, Senior Member, IEEE, Iickho Song, Senior Member, IEEE, \\ Sun Yong Kim, Senior Member, IEEE, and Hyoungmoon Kwon, Student Member, IEEE
}

\begin{abstract}
In this paper, the performance of rake receivers in the presence of fading and impulsive noise is addressed. The optimum maximum likelihood (ML) rake receiver for impulsive fading channel is derived, and a suboptimum rake receiver with a reduced complexity is obtained for practical purposes. Numerical results show that the suboptimum rake receiver exhibits almost the same performance as the optimum rake receiver. It is also observed that, as the number of fingers of a rake receiver increases, the performance of the rake receiver designed for impulsive environment improves, while the rake receiver optimized for Gaussian environment experiences performance degradation in an impulsive environment.
\end{abstract}

Index Terms-Impulsive noise, rake receiver, suboptimum scheme, ultrawideband (UWB), weak signal detection.

\section{INTRODUCTION}

$\mathbf{R}$ ECENT studies [1] in wireless systems indicate that ultrawideband multiple access (UWB-MA) is a viable technology for short-range MA communications. The UWBMA system is characterized by the repetitive transmission of very short pulses (typically in the order of nanosecond) occupying wide frequency bandwidths. For pulse-based UWB-MA systems, multipath components of a signal delayed in time by more than a pulse duration are resolvable [2]. The fine time resolution of multipaths is exploited using a rake receiver to capture a significant amount of energy found in the multipath components and to benefit from a multipath diversity gain.

Most of the rake receivers, including those designed for UWB-MA systems, have been studied and analyzed under the Gaussian noise assumption [2], which is appealing for its analytical tractability and is physically consistent in situations where the central-limit theorem can be invoked. In

Manuscript received December 27, 2004; revised October 20, 2005, January 24, 2006, and January 30, 2006. This work was supported by the Ministry of Science and Technology, Korea, under the National Research Laboratory Program of Korea Science and Engineering Foundation. The review of this paper was coordinated by Prof. C. Tepedelenlioglu.

B. S. Kim is with the Graduate School of Management, Korea Advanced Institute of Science and Technology (KAIST), Seoul 130-722, Korea (e-mail: tree9901@kgsm.kaist.ac.kr).

J. Bae is with the Department of Information and Communication Engineering, Sejong University, Seoul 143-747, Korea (e-mail: baej@sejong.ac.kr).

I. Song and H. Kwon are with the Department of Electrical Engineering, KAIST, Daejeon 305-701, Korea (e-mail: i.song@ieee.org; kwon@ sejong.kaist.ac.kr).

S. Y. Kim is with the Department of Electronics Engineering, Konkuk University, Seoul 143-701, Korea (e-mail: kimsy@kkucc.konkuk.ac.kr).

Digital Object Identifier 10.1109/TVT.2006.878735
UWB-MA systems, however, the sum of the MA interference (MAI) and ambient channel noise [3] can be more adequately modeled as an impulsive interference [4]. The modeling has proved appropriate in many situations including the UWB-MA systems, where the number of users in the communication link is small and the central-limit theorem cannot be applied [5]. When the channel is impulsive, the rake receiver optimized for Gaussian environment, which will be called the Gaussianoptimized (GO) rake receiver in this paper, could experience a severe performance degradation in the UWB-MA systems. This gives rise to the need for redesigning the rake receivers with the impulsive behavior of channel environment taken into account.

This paper attempts to derive and analyze rake receivers in impulsive environment. Under the impulsive interference, we first obtain the optimum rake receiver in the maximumlikelihood (ML) sense. Since the UWB-MA systems transmit signals of extraordinarily small strength with a natural request for low power consumption, it is preferred to design efficient receivers for weak signals in UWB-MA systems. Based on this rationale, we then derive a suboptimum reduced-complexity rake receiver that has an optimum performance at weak signal strengths using the criterion proposed in [6]. The performance of the optimum and suboptimum rake receivers is examined in comparison with that of the GO rake receiver.

The theme and results presented in this paper are clearly different from those in [6], since this paper assumes and addresses the multipath scenario and rake receiver, while Song et al. [6] propose a criterion assuming only a single path. Addressing rake receivers, this paper is distinct also from the studies in [7]-[9], especially in the criterion used for suboptimum schemes and the noise model employed. Another feature of this paper lies in the presentation of some interesting characteristics of the selective rake receiver (SRR) and partial rake receiver (PRR) in impulsive environment.

\section{UWB-MA SYSTEM MODEL}

\section{A. Transmitter Model}

Assume that users employ a binary-phase-shift-keyed (BPSK) modulation with transmitted signals consisting of a low duty-cycle sequence of a large number of UWB pulses. The duration $T_{q}$ of a unit energy UWB pulse $q(t)$ is assumed to be only a very small portion of the frame time (or pulse repetition 
period) $T_{f}$. In this paper, we are not concerned with the shape of the pulses.

The $k$ th user's signal for $0 \leq t \leq N_{s} T_{f}$ is one of the two equiprobable signals

$$
s_{i}^{(k)}(t)=\theta b_{i}^{(k)} \sum_{j=0}^{N_{s}-1} q\left(t-j T_{f}-c_{j}^{(k)} T_{c}\right), \quad i=0,1 .
$$

Here, $N_{s}$ is the number of the UWB pulses modulated by a symbol, $T_{s}=N_{s} T_{f}$ is the symbol duration, $\theta$ is the signal strength, $b_{i}^{(k)}$ is the binary data bit of equiprobable +1 and -1 transmitted by the $k$ th user, $\left\{c_{j}^{(k)}\right\}_{j=0}^{N_{s}-1}$ is the time-hopping sequence of the $k$ th user with period $N_{c}$ (i.e., $0 \leq c_{j}^{(k)} \leq N_{h}$ and $c_{j+n N_{c}}^{(k)}=c_{j}^{(k)}$ for all integers $j$ and $n$ with $N_{h}$ an integer and $c_{j}^{(k)}$ the "location" in the $j$ th frame of the signal of the $k$ th user), and $T_{c}$ is the chip duration.

The frame time $T_{f}$ is chosen to be sufficiently large $\left(T_{f}>N_{h} T_{c}+T_{c}\right)$ to reduce the inter- and intrasymbol interference caused by the delay spread, with the difference $T_{f}-\left(N_{h}+1\right) T_{c}$ called the guard interval. Note that, $N_{c}$ is the theoretical maximum number of users simultaneously active in the UWB-MA system.

\section{B. Channel Model}

Assume the channel model [10] accepted by the IEEE 802.15 Study Group 3a based on indoor channel measurements in the $2-8-\mathrm{GHz}$ frequency band. This model is similar to Saleh-Valenzuela model [11], except that the former employs a log-normal probability density function (pdf) instead of a Rayleigh pdf for the fading-channel coefficients. The fading is assumed to be independent for each cluster and for each ray in a cluster.

The impulse response of the channel is then given by

$$
h(t)=\sum_{m=0}^{M-1} \sum_{n=0}^{N-1} U_{m, n} \delta\left(t-T_{m}-T_{m, n}\right)
$$

where $M$ is the number of clusters, $N$ is the number of multipath components (rays) in a cluster, $U_{m, n}$ is the multipath channel coefficient of the $n$th ray of the $m$ th cluster, $T_{m}$ is the arrival time of the $m$ th cluster, $T_{m, n}$ is the arrival time of the $n$th ray measured from the beginning of the $m$ th cluster, and $\delta(\cdot)$ is the impulse function [12].

The multipath channel coefficient $U_{m, n}$ can be expressed as

$$
U_{m, n}=X_{m, n} V_{m} W_{m, n}
$$

where $X_{m, n}$ is equiprobable +1 and -1 , accounting for the signal inversion due to reflections, $V_{m}$ reflects the fading coefficient associated with the $m$ th cluster, and $W_{m, n}$ corresponds to the fading coefficient associated with the $n$th ray of the $m$ th cluster. The product $V_{m} W_{m, n}$ is a log-normal variable, and the arrival times $\left\{T_{m}\right\}$ of the clusters and arrival times $\left\{T_{m, n}\right\}$ of the rays are both Poisson processes [10]. Without a loss of generality, we assume $T_{0}=0$ and $T_{m, 0}=0$ for $m=1,2, \ldots$
Finally, the pdf of the interarrival time $S_{m}=T_{m}-T_{m-1}$ of clusters is [10]

$$
f_{S_{m}}(t)=\alpha_{c} e^{-\alpha_{c} t}, \quad t \geq 0
$$

and the pdf of the interarrival time $S_{m, n}=T_{m, n}-T_{m, n-1}$ of rays is

$$
f_{S_{m, n}}(t)=\alpha_{r} e^{-\alpha_{r} t}, \quad t \geq 0
$$

where $\alpha_{c}$ and $\alpha_{r}$ are the arrival rates of clusters and rays, respectively.

\section{Receiver Model}

Although the UWB-MA systems are operated over already dedicated frequency bands and consequently encountered narrow- and wideband interference, the effect of such interference upon the system performance is not considered in this paper. Without a loss of generality, we assume that the desired user is $k=1$. The received signal $r(t)$ can then be written as

$$
\begin{aligned}
r(t)= & s_{i}^{(1)}(t) * h(t)+w(t)+n(t) \\
= & \theta b_{i}^{(1)} \sum_{j=0}^{N_{s}-1} \sum_{m=0}^{M-1} \sum_{n=0}^{N-1} U_{m, n} \\
& \cdot q\left(t-j T_{f}-c_{j}^{(1)} T_{c}-T_{m}-T_{m, n}\right)+n^{t}(t)
\end{aligned}
$$

where $*$ is the convolution operation, $w(t)$ represents the MAI caused by other users, $n(t)$ denotes the channel noise, and $n^{t}(t)=w(t)+n(t)$. Note that the MAI $w(t)$ is thought of as a white noise [2] and has been lumped into the channel noise $n(t)$ in (6) to produce the total interference $n^{t}(t)$ modeled as an additive white noise with zero mean.

The received signal $r(t)$ contains $M N$ resolvable multipath components, all of which can be ideally exploited by the all rake receiver [13]. In practice, the number of multipath components utilized in a typical rake combiner is limited by powerconsumption issues, design complexity, and channel estimation. Such issues have motivated studies of multipath combining receivers that process only a subset of the resolvable multipath components.

We consider two such reduced-complexity rake receivers [14], [15]: the SRR and PRR. The SRR selects and combines $L$ strongest multipath channel coefficients among the $M N$ resolvable multipath components. Although the SRR in many cases makes good use of its $L$ fingers available, it requires to keep track of all multipath components. A good tradeoff of the performance degradation versus the receiver complexity is provided by the simpler PRR, which combines $L$ first-arriving paths. As the PRR does not require a selection mechanism, it leads to a substantial complexity reduction in comparison with the SRR.

In this paper, we compare the performance characteristics of the SRR and PRR in impulsive environment. Each of the $L$ paths selected is correlated with the first user's pulse and passed through a matched filter. Assuming that the multipath channel coefficients $\left\{U_{m, n}\right\}$ and the arrival times $\left\{T_{m}\right\}$ and $\left\{T_{m, n}\right\}$ are 
estimated perfectly (e.g., [10]), the output of the matched filter corresponding to the $l$ th finger of the rake receiver at the $j$ th frame is given by

$$
\begin{aligned}
R_{j l} & =\int_{j T_{f}}^{(j+1) T_{f}} r(t) q\left(t-c_{j}^{(1)} T_{c}-t_{j l}\right) d t \\
& =u_{j l} \theta b_{i}^{(1)}+N_{j l}
\end{aligned}
$$

where $N_{j l}=\int_{j T_{f}}^{(j+1) T_{f}} n^{t}(t) q\left(t-c_{j}^{(1)} T_{c}-t_{j l}\right) d t$ is the noise component of the correlator output. In (7), $u_{j l}$ is the estimated value of the $l$ th largest of $\left\{U_{m, n}\right\}$ at the $j$ th frame with $t_{j l}$ as the estimated value of the corresponding arrival time in $\left\{T_{m}+T_{m, n}\right\}$ for the SRR, and $t_{j l}$ is the estimated value of the $l$ th smallest of $\left\{T_{m}+T_{m, n}\right\}$ at the $j$ th frame with $u_{j l}$ as the estimated value of the corresponding multipath channel coefficient in $\left\{U_{m, n}\right\}$ for the PRR.

Denoting by $H_{i}$ the hypothesis that $s_{i}^{(1)}(t)$ is transmitted, the system is now to choose between

$$
H_{0}: R_{j l}=-u_{j l} \theta+N_{j l} \text { and } H_{1}: R_{j l}=u_{j l} \theta+N_{j l}
$$

for $l=0,1, \ldots, L-1$ at the $j$ th frame when the system makes a hard decision per frame.

\section{RAKE RECEIVERS FOR IMPULSIVE ENVIRONMENT}

\section{A. Decision Rules}

Evaluating the likelihood ratio with the two hypotheses $H_{0}$ and $H_{1}$ in (8) and adopting the ML decision rule in the $j$ th frame, the decision region of the hypothesis $H_{i}$ is

$$
D_{i}^{\mathrm{ML}}=\left\{\underline{r}: p_{\underline{R}}\left(\underline{r} \mid s_{i}^{(1)}, \theta\right) \geq p_{\underline{R}}\left(\underline{r} \mid s_{m}^{(1)}, \theta\right), \quad m \neq i\right\}
$$

where $\underline{r}=\left(r_{j 0}, r_{j 1}, \ldots, r_{j, L-1}\right)$, and $p_{\underline{R}}\left(\underline{r} \mid s_{i}^{(1)}, \theta\right)$ represents the conditional pdf of $\underline{R}=\left(R_{j 0}, R_{j 1}, \ldots, R_{j, L-1}\right)$, given that $s_{i}^{(1)}(t)$ is transmitted in the $j$ th frame and the value of the signal strength is $\theta$.

Similarly, using the suboptimum ML (S-ML) decision rule [6], the probability of error is minimized if the decision region of the hypothesis $H_{i}$ is

$$
\begin{aligned}
D_{i}^{\mathrm{S}-\mathrm{ML}}=\{\underline{r}: & \left.\frac{d}{d \theta} p_{\underline{R}}\left(\underline{r} \mid s_{i}^{(1)}, \theta\right)\right|_{\theta=0} \\
& \left.\geq\left.\frac{d}{d \theta} p_{\underline{R}}\left(\underline{r} \mid s_{m}^{(1)}, \theta\right)\right|_{\theta=0}, \quad m \neq i\right\}
\end{aligned}
$$

when the signal strength approaches zero. The S-ML decision rule described by (10) can be derived by using

$$
\left.\frac{\partial P_{e}(\theta)}{\partial \theta}\right|_{\theta=0}=-\frac{1}{2} \sum_{m=1}^{2} \int_{D_{m}}\left\{\left.\frac{\partial}{\partial \theta} p_{\underline{R}}\left(\underline{r} \mid s_{m}^{(1)}, \theta\right)\right|_{\theta=0}\right\} d \underline{r}
$$

in the approximated error probability

$$
P_{e}(\theta) \approx P_{e}(0)+\left.\theta \frac{\partial P_{e}(\theta)}{\partial \theta}\right|_{\theta=0}
$$

where $D_{m}$ denotes the decision region for signal $s_{m}^{(1)}(t)$. A rationale for using the S-ML decision rule is that it is desirable to design a rake receiver, which has an optimum performance at low signal-to-noise ratio (SNR), since the UWB-MA systems are low-power communication systems.

\section{B. Specific Decision Rules in Impulsive Environment}

We first model $\left\{N_{j l}\right\}_{l=0}^{L-1}$ with the independent and identically distributed (i.i.d.) symmetric-alpha-stable $(\mathrm{S} \alpha \mathrm{S})$ distribution [3], which is well known to accurately model impulsive noise processes in many cases. The zero-mean $\mathrm{S} \alpha \mathrm{S}$ pdf is given by

$f_{N_{j l}}(x)= \begin{cases}\frac{1}{\pi} \sum_{k=1}^{\infty} \frac{(-1)^{k-1} \Gamma(k \alpha+1)}{\gamma^{-k} k !|x|^{k \alpha+1}} \cdot \sin \left(\frac{k \alpha \pi}{2}\right), & 0<\alpha \leq 1 \\ \frac{1}{\pi \alpha} \sum_{k=0}^{\infty} \frac{(-1)^{k} x^{2 k}}{(2 k) ! \gamma^{\frac{(2 k+1)}{\alpha}}} \cdot \Gamma\left(\frac{2 k+1}{\alpha}\right), & 1 \leq \alpha \leq 2\end{cases}$

where the positive dispersion parameter $\gamma$ is related to the spread of the $\mathrm{S} \alpha \mathrm{S}$ pdf, and the characteristic exponent $\alpha(0<$ $\alpha \leq 2)$ is related to the heaviness of the tails of the $\mathrm{S} \alpha \mathrm{S}$ pdf. A smaller value of $\alpha$ indicates more severe impulsiveness, while a value closer to two indicates a more Gaussian type of behaviors. The two infinite series in (13) leads to a Cauchy pdf

$$
f_{N_{j l}}(x)=\frac{\gamma}{\pi\left(x^{2}+\gamma^{2}\right)}
$$

when $\alpha=1$, and the second infinite series in (13) produces a Gaussian pdf

$$
f_{N_{j l}}(x)=\frac{1}{2 \sqrt{\gamma \pi}} \exp \left(-\frac{x^{2}}{4 \gamma}\right)
$$

when $\alpha=2$.

Under the Gaussian environment $(\alpha=2)$, we can show that

$$
\sum_{l=0}^{L-1} u_{j l} r_{j l} \underset{H_{0}}{\stackrel{H_{1}}{\gtrless}} 0
$$

using (9) or (10), for which the rake receiver is shown in Fig. 1. The matched filter outputs in Fig. 1 are individually weighted and then combined by a linear combiner called the maximal ratio combining (MRC), providing the maximum output SNR in the Gaussian environment.

When $\alpha=1$, on the other hand, we have the optimum decision rule

$$
\sum_{l=0}^{L-1} \ln \left\{\frac{\left(r_{j l}+u_{j l} \theta\right)^{2}+\gamma^{2}}{\left(r_{j l}-u_{j l} \theta\right)^{2}+\gamma^{2}}\right\} \underset{H_{0}}{\stackrel{H_{1}}{\gtrless}} 0
$$




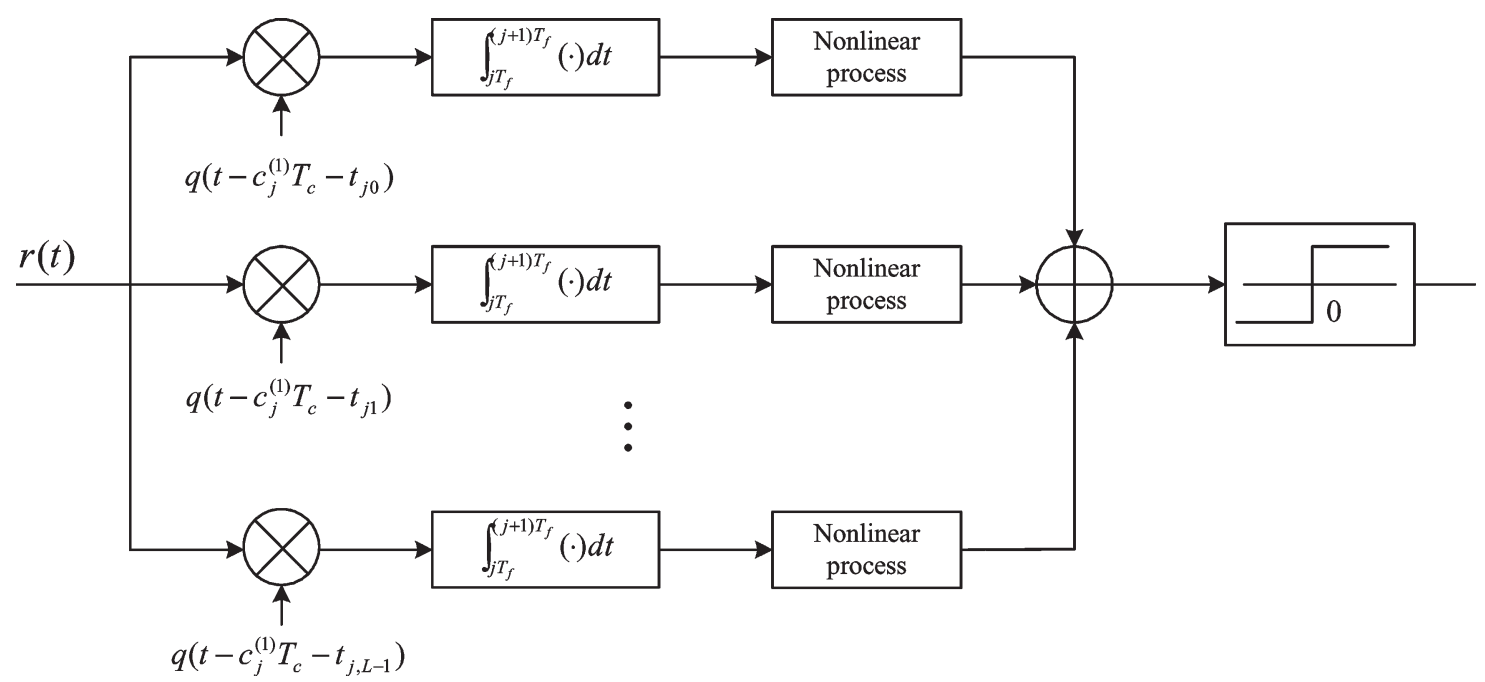

Fig. 1. Rake receiver designed for impulsive environment (when the nonlinear processes are absent, the receiver is for Gaussian environment).

TABLE I

Nonlinear Processes for Some Distributions [SGN(·) Denotes the Signum Function]

\begin{tabular}{|c|cc|}
\hline distribution & ML rake receiver & S-ML rake receiver \\
\hline \hline generalized Gaussian & $\left|r_{j l}+u_{j l} \theta\right|^{k}-\left|r_{j l}-u_{j l} \theta\right|^{k}$ & $u_{j l}\left|r_{j l}\right|^{k-1} \operatorname{sgn}\left(r_{j l}\right)$ \\
\hline generalized Cauchy & $\ln \left(\frac{v A_{G}^{k}(k)+\left|r_{j l}+u_{j l} \theta\right|^{k}}{v A_{G}^{k}(k)+\left|r_{j l}-u_{j l} \theta\right|^{k}}\right)$ & $\frac{u_{j l}\left|r_{j l}\right|^{k-1} \operatorname{sgn}\left(r_{j l}\right)}{v A_{G}^{k}(k)+\left|r_{j l}\right|^{k}}$ \\
\hline$t$ & $\ln \left(\frac{\left(r_{j l}+u_{j l} \theta\right)^{2}+n}{\left(r_{j l}-u_{j l} \theta\right)^{2}+n}\right)$ & $\frac{u_{j l} r_{j l}}{r_{j l}^{2}+n}$ \\
\hline logistic & $\ln \left(\frac{e^{\rho \theta u_{j l} l}+e^{-\rho r_{j l}}}{1+e^{-\rho\left(r_{j l}-u_{j l} \theta\right)}}\right)$ & $u_{j l}\left(\frac{1-e^{-\rho r_{j l}}}{1+e^{-\rho r_{j l}}}\right)$ \\
\hline
\end{tabular}

using (9), and the suboptimum decision rule

$$
\sum_{l=0}^{L-1} \frac{u_{j l} r_{j l}}{r_{j l}^{2}+\gamma^{2}} \underset{H_{0}}{\stackrel{H_{1}}{\gtrless}} 0
$$

from (10), for which the rake receivers are also as shown in Fig. 1. The rake receiver based on (17), which is called the Cauchy-optimized $(\mathrm{CO})$ rake receiver, requires an estimate of the signal strength $\theta$, while the rake receiver based on (18), which is called the Cauchy-suboptimized (CS) rake receiver does not. This implies that the $\mathrm{CS}$ rake receiver requires a less structural complexity than the $\mathrm{CO}$ rake receiver. We shall see later that the performance difference between the $\mathrm{CO}$ and $\mathrm{CS}$ rake receivers is insignificant.

Unlike the GO rake receiver based on (16), the rake receiver for impulsive noise employs a nonlinear process at each finger before the observations are summed. The nonlinear process in Fig. 1 is $\ln \left[\left\{\left(r_{j l}+u_{j l} \theta\right)^{2}+\gamma^{2}\right\} /\left\{\left(r_{j l}-u_{j l} \theta\right)^{2}+\gamma^{2}\right\}\right]$ and $\left(u_{j l} r_{j l}\right) /\left(r_{j l}^{2}+\gamma^{2}\right)$ for the CO and CS rake receivers, respectively, as is evident from (17) and (18). The nonlinear process reduces the influence of observations with very large amplitudes, resulting in the performance stability in impulsive environment as reported in robust signal-detection theory [16], [17]. The dispersion parameter $\gamma$ in the $\mathrm{CO}$ and $\mathrm{CS}$ rake receivers can be estimated easily by using only the sample mean and variance of independent realizations of the S $\alpha$ S process [18]. From now on, we will focus on the $\mathrm{CO}$ and $\mathrm{CS}$ rake receivers, since detectors for a Cauchy environment exhibit [8] desirable performance characteristics under various impulsive environment.

For the generalized Gaussian, generalized Cauchy, $t-$, and logistic distributions having the pdfs

$$
\begin{aligned}
f_{\mathrm{GG}}(x) & =\frac{k}{2 A_{G}(k) \Gamma(1 / k)} \cdot \exp \left\{-\left(\frac{|x|}{A_{G}(k)}\right)^{k}\right\}, \quad k>0 \\
f_{\mathrm{GC}}(x) & =\frac{\tilde{B}_{c}(k, v)}{\tilde{D}_{c}^{v+1 / k}(x)}, \quad k>0, v>0 \\
f_{T}(x) & =\frac{\Gamma((n+1) / 2)}{\sqrt{\pi n} \Gamma(n / 2)} \cdot\left(1+\frac{x^{2}}{n}\right)^{-\frac{n+1}{2}}, \quad n>0
\end{aligned}
$$

and

$$
f_{L}(x)=\frac{\rho e^{-\rho x}}{\left(1+e^{-\rho x}\right)^{2}}, \quad \rho>0
$$

respectively, the nonlinear processes for the ML and S-ML rake receivers are tabulated in Table $\mathrm{I}$, which can be obtained from (9) and (10) after some manipulations. In (19)- $-(22), \Gamma(a)$ is the gamma function

$$
A_{G}(k)=\sqrt{\frac{\sigma_{G}^{2} \Gamma(1 / k)}{\Gamma(3 / k)}}
$$


with $\sigma_{G}^{2}$ the variance

$$
\tilde{B}_{c}(k, v)=\frac{k v^{k} \Gamma\left(v+\frac{1}{k}\right)}{2 A_{G}(k) \Gamma(v) \Gamma(1 / k)}
$$

and

$$
\tilde{D}_{c}(x)=1+\frac{1}{v}\left\{\frac{|x|}{A_{G}(k)}\right\}^{k}
$$

The generalized Gaussian pdf (19) becomes the double exponential (Laplace) pdf $f_{D}(x)=(\lambda) /(2) e^{-\lambda|x|}, \quad \lambda>0$ when $k=1$.

\section{Numerical Results}

Due to the infinite variance of the $\mathrm{S} \alpha \mathrm{S}$ noise when $\alpha<2$, we employ the geometric SNR (G-SNR) [19] to indicate the relative strength between the information-bearing signal and $\mathrm{S} \alpha \mathrm{S}$ noise. The geometric power $S_{0}$ of an $\mathrm{S} \alpha \mathrm{S}$ variable is defined as

$$
S_{0}=\frac{\left(C_{g} \gamma\right)^{1 / \alpha}}{C_{g}}
$$

where

$$
\begin{aligned}
C_{g} & =\exp \left\{-\lim _{s \rightarrow \infty}\left(\ln s-\sum_{z=1}^{s} \frac{1}{z}\right)\right\} \\
& \approx 1.78
\end{aligned}
$$

is the exponential of the Euler constant. The G-SNR is then given as

$$
\mathrm{G}-\mathrm{SNR}=\frac{1}{2 C_{g}}\left(\frac{\theta}{S_{0}}\right)^{2}
$$

which becomes the standard SNR when $\alpha=2$.

Assuming that the fading is sufficiently slow so that a large number of bits are transmitted over essentially the same channel [13], the performance of the SRR and PRR is evaluated and compared through Monte Carlo simulations. The Monte Carlo simulations are set up based on the channel model one described in [10], and the bit error rate is obtained from $2.5 \times 10^{6}$ Monte Carlo runs, where noise samples $\left\{N_{j l}\right\}_{l=0}^{L-1}$ are generated using

$$
\gamma^{\frac{1}{\alpha}} \frac{\sin \alpha}{(\cos A)^{1 / \alpha}}\left[\frac{\cos \{(1-\alpha) A\}}{B}\right]^{\frac{1-\alpha}{\alpha}} .
$$

In (29), $A$ is uniform on $(-\pi / 2, \pi / 2)$ and $B$ is exponential with mean one [3], [18]. Without a loss of generality, we assume $\gamma=1$.

\section{A. Performance of the SRR in Impulsive Environment}

Figs. 2-5 show the performance characteristics of the GO, CO, and CS SRRs, assuming that the $L$ largest multipath gains are given or have already been estimated accurately. In the

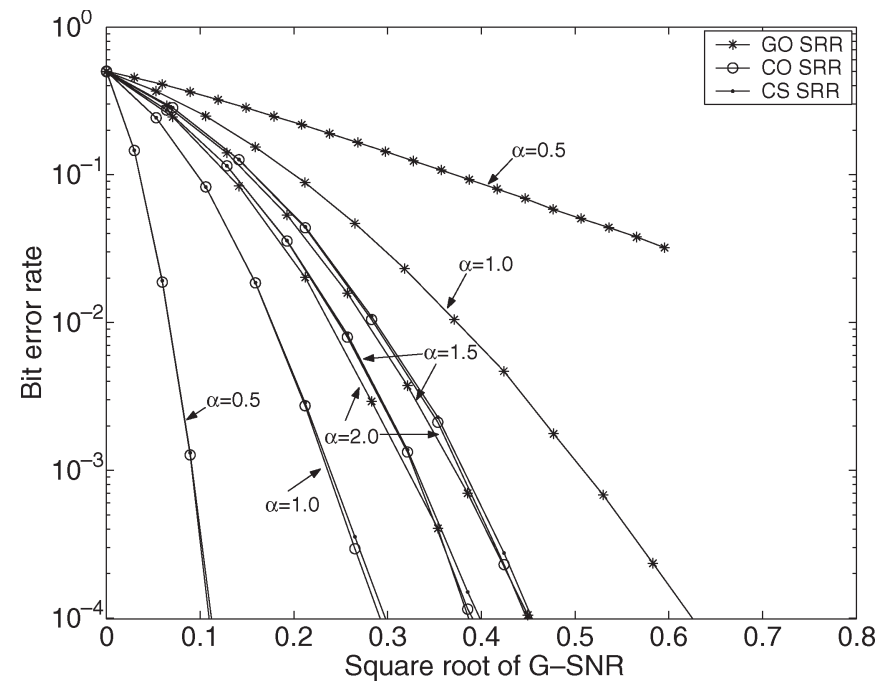

Fig. 2. Performance characteristics of the GO, CO, and CS SRRs with $L=6$ when $N_{s}=100$ in the $\mathrm{S} \alpha \mathrm{S}$ environment with $\alpha=0.5, \alpha=1.0, \alpha=1.5$, and $\alpha=2.0$.



Fig. 3. Performance characteristics of the GO, CO, and CS SRRs with $L=3$ and 6 in the $\mathrm{S} \alpha \mathrm{S}$ environment with $\alpha=2$.

interpretation of the figures, it is to be recalled that although we have shown several graphs in one figure due to space constraint, comparison of the same detector for different values of $\alpha$ (comparison of the GO SRR for $\alpha=0.5$ with that for $\alpha=1$ in Fig. 2, for example) is meaningless, since the noise power in the usual sense is not defined or applicable for the $\mathrm{S} \alpha \mathrm{S}$ noise, and the signal strength varies as the value of $\alpha$ when the G-SNR is fixed. For example, from (26) and (28), the relationships $\theta_{1} \approx \theta_{0.5} / 1.78, \theta_{1.5} \approx \theta_{1} / 1.21$, and $\theta_{2} \approx \theta_{1.5} / 1.10$ are required to result in the same G-SNR when $\gamma=1$, where $\theta_{p}$ denotes the signal strength when $\alpha=p$.

It is observed that the CO and CS SRRs possess almost the same performance characteristics especially when the G-SNR is small. The CO and CS SRRs uniformly outperform the GO SRR in impulsive environment with the outperformance becoming clearer as the impulsiveness gets higher ( $\alpha$ gets smaller), as is evident in Fig. 2, for example. The performance 


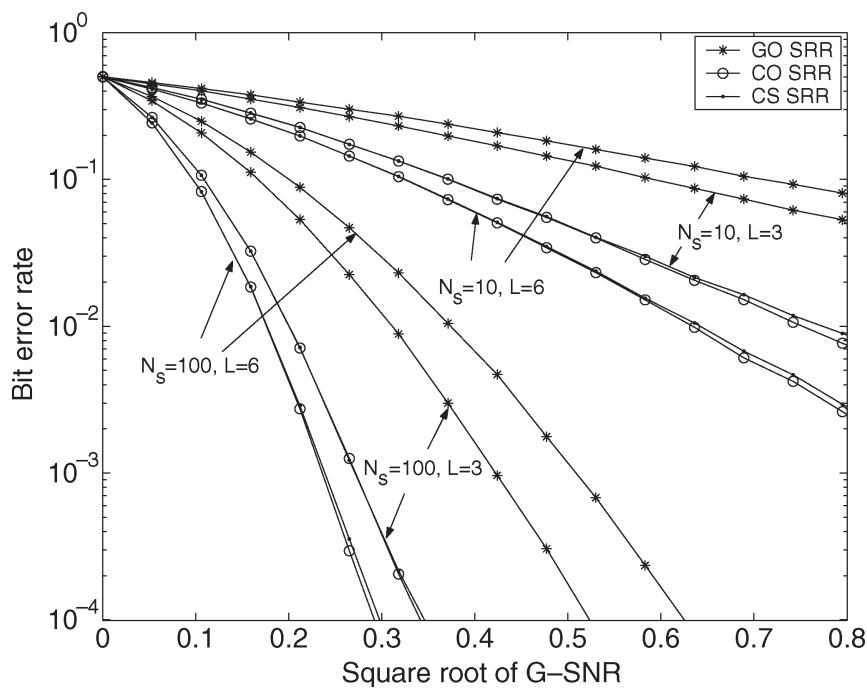

Fig. 4. Performance characteristics of the GO, CO, and CS SRRs with $L=3$ and 6 in the $\mathrm{S} \alpha \mathrm{S}$ environment with $\alpha=1$.

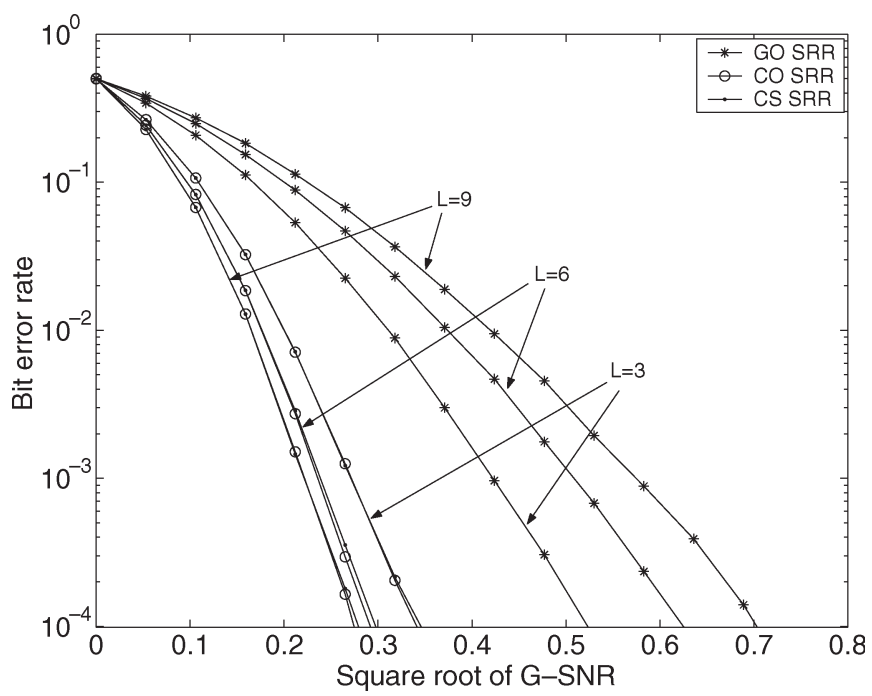

Fig. 5. Performance characteristics of the GO, CO, and CS SRRs with $L=3$, $L=6$, and $L=9$ when $N_{s}=100$ in the $\mathrm{S} \alpha \mathrm{S}$ environment with $\alpha=1$.

of all the three SRRs generally improves when the number $L$ of the fingers of the SRR increases in the Gaussian environment (Fig. 3) and when the number $N_{s}$ of the UWB pulses per symbol increases (Figs. 3 and 4 ).

It is interesting to note that the GO SRR performs worse as the number $L$ increases when the interference is impulsive (Figs. 4 and 5), while the CO and CS SRRs perform better as the number $L$ increases irrespective of the interference distribution (Figs. 3-5). Again, this result is not unexpected from the signaldetection point of view: It is well known [8], [17] that an observation with a very large magnitude in impulsive environment should be considered not as a signal plus noise but as noise only. The nonlinear processes in Fig. 1 attenuate the influence of observations with very large magnitudes, thereby protecting the $\mathrm{CO}$ and CS SRRs from using the observations inappropriately. On the other hand, the GO SRR favors any observation with a larger magnitude, resulting in a poor performance in nonGaussian environment.

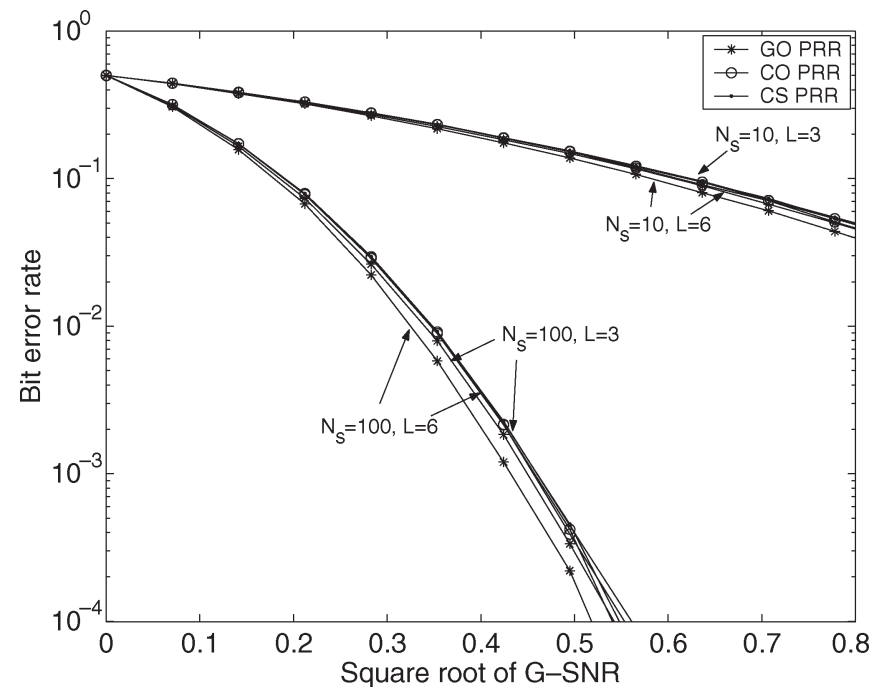

Fig. 6. Performance characteristics of the GO, CO, and CS PRRs with $L=3$ and 6 in the $\mathrm{S} \alpha \mathrm{S}$ environment with $\alpha=2$.

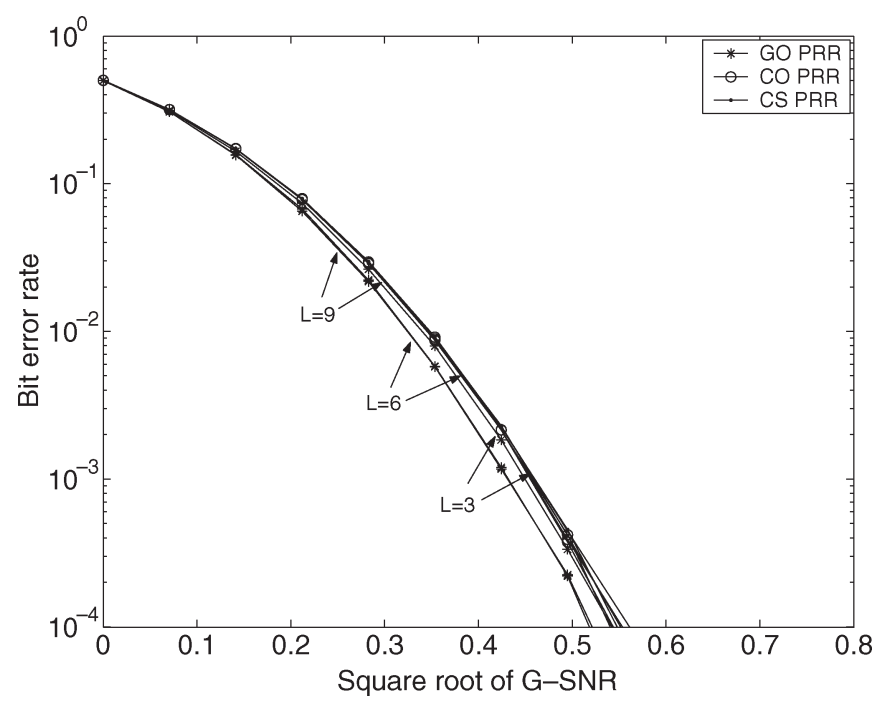

Fig. 7. Performance characteristics of the GO, CO, and CS PRRs with $L=3$, $L=6$, and $L=9$ when $N_{s}=100$ in the S $\alpha$ S environment with $\alpha=2$.

\section{B. Performance of the PRR in Impulsive Environment}

A selection of results, showing the performance characteristics of the GO, CO, and CS PRRs, is given in Figs. 6 and 7, on which we can make observations similar to those on Figs. 2-5 for SRRs. Two points are noticeable here. First, the number $L$ of the fingers affects less on the performance of the PRRs than on that of the SRRs. Second, unlike the CO and CS SRRs (Fig. 3), the CO and CS PRRs have nearly the same performance as the GO PRR, even in the Gaussian environment. This is because the outputs $\left\{r_{j l}\right\}$ of the matched filters in the PRR tend to be smaller than those in the SRR, resulting in $\left(r_{j l} u_{j l}\right) /\left(r_{j l}^{2}+1\right) \approx r_{j l} u_{j l}$.

\section{Comparison of the SRR and PRR}

Fig. 8 exhibits the performance difference between the SRR and PRR in a more direct way. The GO SRR is reported 


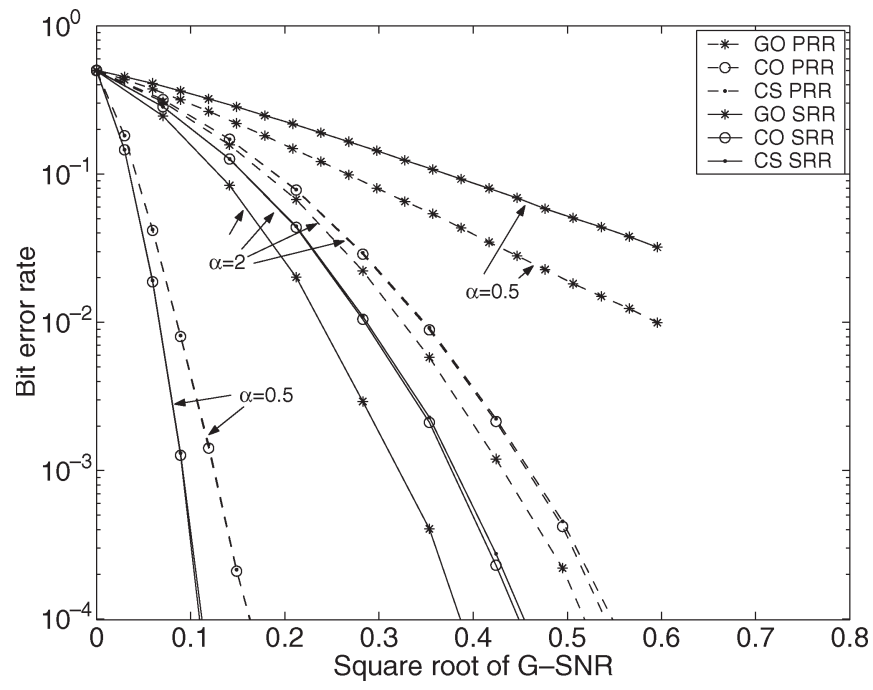

Fig. 8. Performance characteristics of the GO, CO, and CS PRRs and SRRs with $L=6$ when $N_{s}=100$ in the $\mathrm{S} \alpha \mathrm{S}$ environment with $\alpha=0.5$ and $\alpha=2.0$.



Fig. 9. Performance characteristics of the GO, CO, and CS SRRs with $L=6$ when $N_{s}=50$ in the Cauchy environment.

[15] to perform better than the GO PRR in Gaussian environment if the multipath gains are known accurately. In impulsive noise, on the contrary, the GO SRR performs worse than the GO PRR. This is because, the GO SRR, not having the nonlinear processes, tends to favor observations with large magnitudes, which have probably been contaminated by the impulsive channel noise, and thus, the GO SRR is influenced more adversely by the impulsive environment than the GO PRR.

On the other hand, as we have mentioned previously, the $\mathrm{CO}$ and CS SRRs limit the influence of the impulsive channel noise with the nonlinear process and consequently perform better than the PRR in the Gaussian as well as impulsive environment. Finally, let us just add that the CS and CO rake receivers with $\gamma$ estimated inaccurately (to a certain degree) have almost the same performance as those with the accurate $\gamma$, especially at small signal strength, as shown in Figs. 9 and 10.

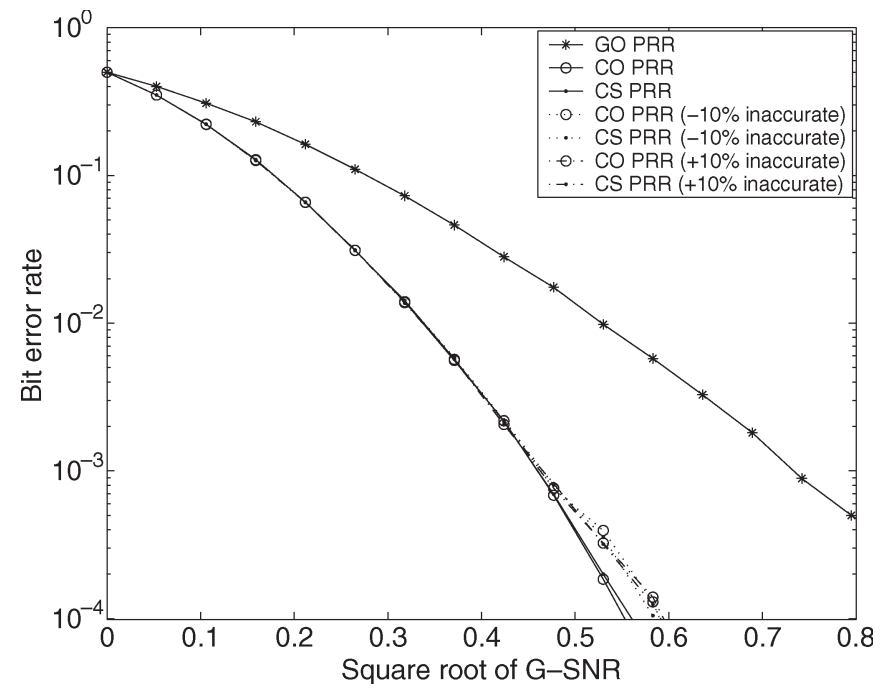

Fig. 10. Performance characteristics of the GO, CO, and CS PRRs with $L=6$ when $N_{s}=50$ in the Cauchy environment.

\section{CONCLUding REMARK}

The optimum and suboptimum rake receivers have been derived based on the ML and S-ML decision rules, respectively, in an impulsive environment. The $\mathrm{CO}$ and $\mathrm{CS}$ rake receivers generally outperform the GO rake receiver in impulsive environment. The performance of the CS rake receiver differs barely from that of the $\mathrm{CO}$ rake receiver despite the CS rake receiver being of simpler structure than the $\mathrm{CO}$ rake receiver. As the number of fingers increases in impulsive environment, the performance of the $\mathrm{CO}$ and $\mathrm{CS}$ rake receivers improves, while that of the GO rake receiver degrades. The CO and CS SRRs perform better than the CO and CS PRRs, while the GO SRR performs worse than the GO PRR in an impulsive environment.

Although the rake receivers derived in this paper for an impulsive environment have been considered in the UWB-MA system for convenience, rake receivers can also be applied in other communication systems, such as direct-sequence codedivision MA (DS-CDMA) and wideband CDMA (WCDMA).

\section{ACKNOWLEDGMENT}

The authors would like to thank the anonymous reviewers for their constructive suggestions and comments, with which the authors have improved the readability and technical contents of this paper.

\section{REFERENCES}

[1] E. Baccarelli, M. Biagi, C. Pelizzoni, and P. Bellotti, "A novel multiantenna impulse radio UWB transceiver for broadband high-throughput 4G WLANs," IEEE Commun. Lett., vol. 8, no. 7, pp. 419-421, Jul. 2004.

[2] V. Lottici, A. Andrea, and U. Mengali, "Channel estimation for ultrawideband communications," IEEE J. Sel. Areas Commun., vol. 20, no. 9 , pp. 1638-1645, Dec. 2002.

[3] G. A. Tsihrintzis and C. L. Nikias, "Incoherent receiver in alphastable impulsive noise," IEEE Trans. Signal Process., vol. 43, no. 9, pp. 2225-2229, Sep. 1995.

[4] S. Yoon, I. Song, and S. Y. Kim, "Code acquisition for DS/SS communications in non-Gaussian impulsive channels," IEEE Trans. Commun., vol. 52, no. 2, pp. 187-190, Feb. 2004. 
[5] B. Hu and N. C. Beaulieu, "Accurate evaluation of multiple-access performance in TH-PPM and TH-BPSK UWB systems," IEEE Trans. Commun., vol. 52, no. 10, pp. 1758-1766, Oct. 2004.

[6] I. Song, J. K. Koo, H. Kwon, S. R. Park, S. R. Lee, and B.-H. Chung, "A novel detection criterion for weak $M$-ary signals and its application to ultra wideband multiple access systems," IEEE Trans. Veh. Technol., vol. 54, no. 6, pp. 2024-2036, Nov. 2005.

[7] A. D. Spaulding, "Locally optimum and suboptimum detector performance in a non-Gaussian interference environment," IEEE Trans. Commun., vol. COM-33, no. 6, pp. 509-517, Jun. 1985.

[8] S. A. Kassam, Signal Detection in Non-Gaussian Noise. New York: Springer-Verlag, 1988.

[9] G. A. Tsihrintzis and C. L. Nikias, "Performance of optimum and suboptimum receivers in the presence of impulsive noise modeled as an alphastable process," IEEE Trans. Commun., vol. 43, no. 234, pp. 904-914, Feb.-Apr. 1995.

[10] A. F. Molisch, J. R. Foerster, and M. Pendergrass, "Channel models for ultrawideband personal area networks," IEEE Wireless Commun., vol. 10, no. 6, pp. 14-21, Dec. 2003 .

[11] A. Saleh and R. Valenzuela, "A statistical model for indoor multipath propagation," IEEE J. Sel. Areas Commun., vol. SAC-5, no. 2, pp. 128-137, Feb. 1987.

[12] R. P. Kanwal, Generalized Functions: Theory and Technique. Basel, Switzerland: Birkhäuser, 1997.

[13] M. Z. Win and R. A. Scholtz, "Characterization of ultra-wide bandwidth wireless indoor channels: A communication-theoretic view," IEEE J. Sel. Areas Commun., vol. 20, no. 9, pp. 1613-1627, Dec. 2002.

[14] G. E. Bottomley, T. Ottosson, and Y.-P. E. Wang, "A generalized rake receiver for interference suppression," IEEE J. Sel. Areas Commun., vol. 18 , no. 8, pp. 1536-1545, Aug. 2000.

[15] A. F. Molisch, M. Z. Win, and J. H. Winters, "Reduced-complexity transmit/receive-diversity systems," IEEE Trans. Signal Process., vol. 51, no. 11, pp. 2729-2738, Nov. 2003.

[16] D. J. Warren and J. B. Thomas, "Asymptotically robust detection and estimation for very heavy-tailed noise," IEEE Trans. Inf. Theory, vol. 37, no. 3, pp. 475-481, May 1991.

[17] I. Song, J. Bae, and S. Y. Kim, Advanced Theory of Signal Detection. New York: Springer-Verlag, 2002.

[18] R. Kapoor, A. Banerjee, G. A. Tsihrintzis, and N. Nandhakumar, "UWB radar detection of targets in foliage using alpha-stable clutter models," IEEE Trans. Aerosp. Electron. Syst., vol. 35, no. 3, pp. 819-834, Jul. 1999.

[19] T. C. Chuah, B. S. Sharif, and O. R. Hinton, "Nonlinear decorrelator for multiuser detection in non-Gaussian impulsive environments," Electron. Lett., vol. 36, no. 10, pp. 920-922, May 2000.

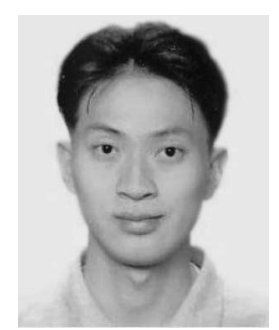

Byoung Soo Kim was born in Kwangju, Korea, in 1980. He received the B.S.E. degree in electrical engineering from Korea University, Seoul, Korea, in 2003 and the M.S.E. degree in electrical engineering from Korea Advanced Institute of Science and Technology (KAIST), Daejeon, Korea, where he is currently working toward the Ph.D. degree at the Graduate School of Management.

Since March 2005, he has been a Research Assistant with the Graduate School of Management, KAIST. His research interests are in mobile communications policy and digital economics.

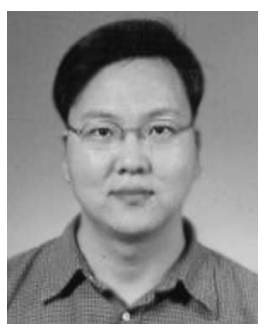

Jinsoo Bae (S'93-M'98-SM'03) was born in Seoul, Korea, in 1972. He received the B.S.E. (summa cum laude), M.S.E., and Ph.D. degrees in electrical engineering from Korea Advanced Institute of Science and Technology (KAIST), Daejeon, Korea, in 1993, 1995, and 1998, respectively.

$\mathrm{He}$ is currently an Associate Professor with the Department of Information and Communication Engineering, Sejong University, Seoul. His research interests include signal-detection theory, digital signal processing, and channel-coding theory.

Dr. Bae is a member of Korean Institute of Communication Sciences (KICS) and Institute of Electronics, Information, and Communication Engineers (IEICE).

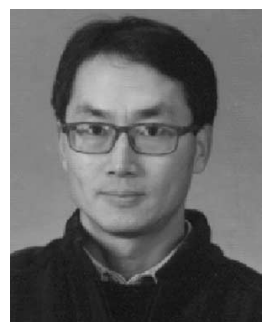

Iickho Song (S'80-M'82-SM'96) was born in Seoul, Korea, in 1960. He received the B.S.E. (magna cum laude) and M.S.E. degrees in electronics engineering from Seoul National University, Seoul, in 1982 and 1984, respectively, and the M.S.E. and $\mathrm{Ph} . \mathrm{D}$. degrees in electrical engineering from the University of Pennsylvania, Philadelphia, in 1985 and 1987, respectively.

In 1987, he was a member of the technical staff with Bell Communications Research. In 1988, he joined the Department of Electrical Engineering, Korea Advanced Institute of Science and Technology (KAIST), Daejeon, Korea, where he is currently a Professor. He has coauthored Advanced Theory of Signal Detection (Springer, 2002) and Random Processes (in Korean, Saingneung, 2004), and has published a number of papers on signal detection and mobile communications. His research interests include detection and estimation theory, statistical theory of communication and signal processing, and mobile communications.

Dr. Song served as the Treasurer of the IEEE Korea Section in 1989. He has received many awards, including the Young Scientists Award presented by the President of the Republic of Korea in 2000.

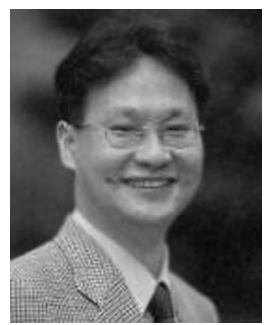

Sun Yong Kim (S'91-M'96-SM'01) was born in Incheon, Korea, in 1968. He received the B.S.E. (summa cum laude), M.S.E., and Ph.D. degrees in electrical engineering from Korea Advanced Institute of Science and Technology (KAIST), Daejeon, Korea, in 1990, 1993, and 1995, respectively.

From April 1995 to March 1996, he was a Visiting Researcher with University of Tokyo, Tokyo, Japan. From March 1996 to August 2001, he was with the Department of Electronics Engineering, Hallym University, Chuncheon, Korea. Since September 2001, he has been with the Department of Electronics Engineering, Konkuk University, Seoul, Korea, where he is currently an Associate Professor. His research interests include detection and estimation theory, statistical signal processing, and analysis and design of mobile communication systems.

$\mathrm{He}$ is a member of the Korean Institute of Communication Sciences (KICS) and the Institute of Electronics Engineers of Korea (IEEK). He received the Second-Best Paper Award from the IEEE Korea Section in 1990, a scholarship from the IEEE Communication Society in 1992-1993, and a Paper Award from the LG Information and Communications in 1994.

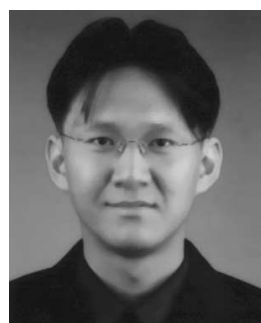

Hyoungmoon Kwon (S'00) received the B.S.E. degree in electronics engineering from Yonsei University, Seoul, Korea, in 2000 and the M.S.E. degree in electrical engineering from Korea Advanced Institute of Science and Technology (KAIST), Daejeon, Korea, where he is currently working toward the Ph.D. degree.

Since March 2000, he has been a Teaching and Research Assistant with the Department of Electrical Engineering, KAIST. His research interests include spread-spectrum systems and detection theory. 